\title{
Budget impact of lasmiditan for the acute treatment of migraine in the United States
}

\author{
Sandra Milev, MS; Gerhardt Pohl, PhD; Ariel Sun, MA; Oksana Mason, MA;
}

Nancy Njuguna, BPharm, MBA; and Li Shen Loo

\section{What is already known about this subject}

- In 2019 and 2020, the FDA approved 3 novel acute treatments for migraine- lasmiditan, rimegepant, and ubrogepant-for adults with migraine with and without aura.

- Unlike triptans, these treatments are not contraindicated in patients with cardiovascular conditions.

- These medications represent an increase in cost of treatment above that of generic oral triptans.

\section{What this study adds}

- This study performed a detailed analysis of potential budget impact of the novel acute treatments for migraine based on US prices and details of usage from the respective US labels.

- Costs in the broad population indicated in the US label were compared with a narrower population recommended in an American Headache Society consensus statement.

- The study also compares the budget implications of benefit designs requiring previous steps through 1 vs 2 oral triptans.

with migraine. A model with a 3-year time horizon was built assuming that demand for the novel acute treatments would not vary based on whether lasmiditan is included in the formulary. The model examined a variety of populations, in particular beneficiaries with previous use of 1 or more oral triptans or contraindicated to triptans and beneficiaries with previous use of 2 or more oral triptans or contraindicated to triptans. Primary outcomes were the incremental differences in total cost and average cost per member per month (PMPM) between scenarios with and without lasmiditan. One-way sensitivity analyses with model parameters that were varied by plus or minus $15 \%$ were conducted to assess the effect of key parameters on the incremental total cost over 3 years.

RESULTS: The addition of lasmiditan to a formulary that already includes ubrogepant and rimegepant resulted in a total savings of $-\$ 927,657$ ( $-1.5 \%$ compared with the scenario without lasmiditan) over a 3-year

\section{Author affiliations}

Sandra Milev, MS, and Ariel Sun, MA, Evidera, San Francisco, CA. Gerhardt Pohl, PhD; Oksana Mason, MA; Nancy Njuguna, BPharm, MBA; and Li Shen Loo, Eli Lilly and Company, Indianapolis, IN.

AUTHOR CORRESPONDENCE:

Gerhardt Pohl, 317.801.2182;

gerhardt_pohl@lilly.com

J Manag Care Spec Pharm 2021;27(12):1714-23

Copyright $@ 2021$, Academy of Managed Care Pharmacy. All rights reserved.

time horizon in the population with previous history of using 1 or more oral triptans or contraindicated to a triptan. In the population with previous history of using 2 or more oral triptans or contraindicated, the addition of lasmiditan resulted in a total budget impact of $-\$ 466,518(-1.3 \%)$ over a 3-year time horizon. Most of the cost savings was attributable to reductions in drug acquisition cost. Savings in total costs resulted in average incremental cost per PMPM of -0.03 and $-\$ 0.01$, respectively.

CONCLUSIONS: The addition of lasmiditan to the formulary as a novel acute treatment option for migraine alongside ubrogepant and rimegepant resulted in lower budget impact on a 3-year time horizon from a US commercial payer's perspective. This result is important to US commercial payers as they seek to incorporate the emerging novel acute treatments for migraine into their benefit designs. 
Migraine is a chronic neurologic disease typically characterized by moderate or severe throbbing headache pain; is often associated with photophobia and phonophobia, nausea, and/or vomiting; and can be exacerbated by physical activity. ${ }^{1}$ Migraine may be preceded by aura, a preliminary symptom manifesting as visual, sensory, or other central nervous system symptoms that are then followed by primary migraine symptoms. ${ }^{1}$ It is estimated that migraine affects $11.6 \%$ of the global population. ${ }^{2}$ The prevalence of migraine is significantly higher in women than in men $(13.8 \%$ vs $6.9 \%$, respectively). ${ }^{2}$ In the United States alone, migraine affects roughly 1 in 7 adults, 1 in 4 households, and more than 36 million individuals. ${ }^{1,3-6}$

Migraine is associated with high levels of humanistic and economic burden in terms of reduced health-related quality of life and increased medical costs (direct and indirect). ${ }^{7}$ Annual direct health care expenditures associated with migraine in the United States are estimated at \$56.31 billion, ${ }^{8}$ and the annual indirect costs (ie, lost productivity, absenteeism, and short-term disability) can range widely from $\$ 943$ per person for episodic and $\$ 3,300$ for chronic migraine. ${ }^{9,10}$

A goal of acute treatment of migraine is to alleviate attacks rapidly and consistently without recurrence and to minimize the use of rescue medications. ${ }^{11}$ Current pharmacotherapies recommended by the American Academy of Neurology and the American Headache Society (AHS) include analgesics, ergots, nonsteroidal anti-inflammatory drugs, opioids (butorphanol nasal spray $1 \mathrm{mg}$ ), and triptans..$^{12}$ The American Migraine Prevalence and Prevention (AMPP) study found that $41 \%$ of patients treated for episodic migraine had at least 1 unmet treatment need from among treatment dissatisfaction, moderate or severe migraine-related disability, excessive use of opioids or barbiturates, recurrent use of emergency department, or a history of cardiovascular events indicating a possible contradiction to triptan use. ${ }^{13}$ While triptans are efficacious for the acute treatment of migraine, up to $40 \%$ of attacks (and 30\% of patients) fail to respond to a particular triptan. ${ }^{14,15}$ Triptans are also associated with peripheral vasoconstriction due to effects mediated by activation of (5HT) 1B receptors and are, therefore, contraindicated in people with cardiovascular risks.

Three novel compounds have been approved by the US Food and Drug Administration in the past year for the acute treatment of migraine with and without aura in adults. The AHS position statement recommends consideration of these emerging novel acute treatments for patients who have contraindications to the use of triptans or failed to respond to or tolerate oral triptans, as determined by either a validated patient-reported outcome questionnaire (such as the Migraine Treatment Optimization Questionnaire [mTOQ] or other instrument) or health care provider attestation. ${ }^{16}$ Lasmiditan, the first oral selective serotonin (5HT) $1 \mathrm{~F}$ receptor agonist, was approved in October 2019. ${ }^{17}$ Ubrogepant and rimegepant, both oral calcitonin gene-related peptide (CGRP) receptor antagonists, received approval in December 2019 and February 2020, respectively. ${ }^{18,19}$ The near simultaneous approval of these medications complicates budget impact modeling, since in practice, plans may consider a variety of subsets of the 3 medications for their formularies.

For simplicity, we compared scenarios that partitioned based on the 2 mechanistic classes of drugs: selective serotonin (5HT) $1 \mathrm{~F}$ receptor agonist and oral CGRP receptor agonists. We did not distinguish uptake or usage between the 2 oral CGRP receptor agonists other than with respect to potential second doses, which are excluded from the US label for rimegepant but permitted for ubrogepant.

This study aimed to estimate the budget impact of adopting lasmiditan alongside the other novel acute treatments ubrogepant and rimegepant from a third-party commercial payer perspective in the United States.

\section{Methods}

\section{MODEL OVERVIEW}

A decision-analytic economic model was developed to evaluate the costs of including lasmiditan as an acute treatment option for adults with migraine in the United States. For convenience in adapting results to various actual plan sizes, the displayed model calculated the number of patients who are eligible to receive novel acute treatments within a hypothetical plan with 1 million members. Patients were allocated to different treatment options each year based on the estimated market share data. Market share for other treatment options decreased with a corresponding uptake in market share of novel acute treatments. The overall cost of the market mix was calculated based on the drug unit costs and administration costs (when applicable) for each treatment, as well as the treatment's dosing regimen and annual frequency of use.

A scenario that reflected current and new treatments in the market without lasmiditan was compared with one that included lasmiditan. Growth in total market share for the novel acute treatments was assumed to remain the same in both scenarios whether or not lasmiditan was included on formulary. The budget impact to a payer was the difference in projected expenditures between the 2 scenarios. 


\section{FIGURE 1 Size of Eligible Target Population}

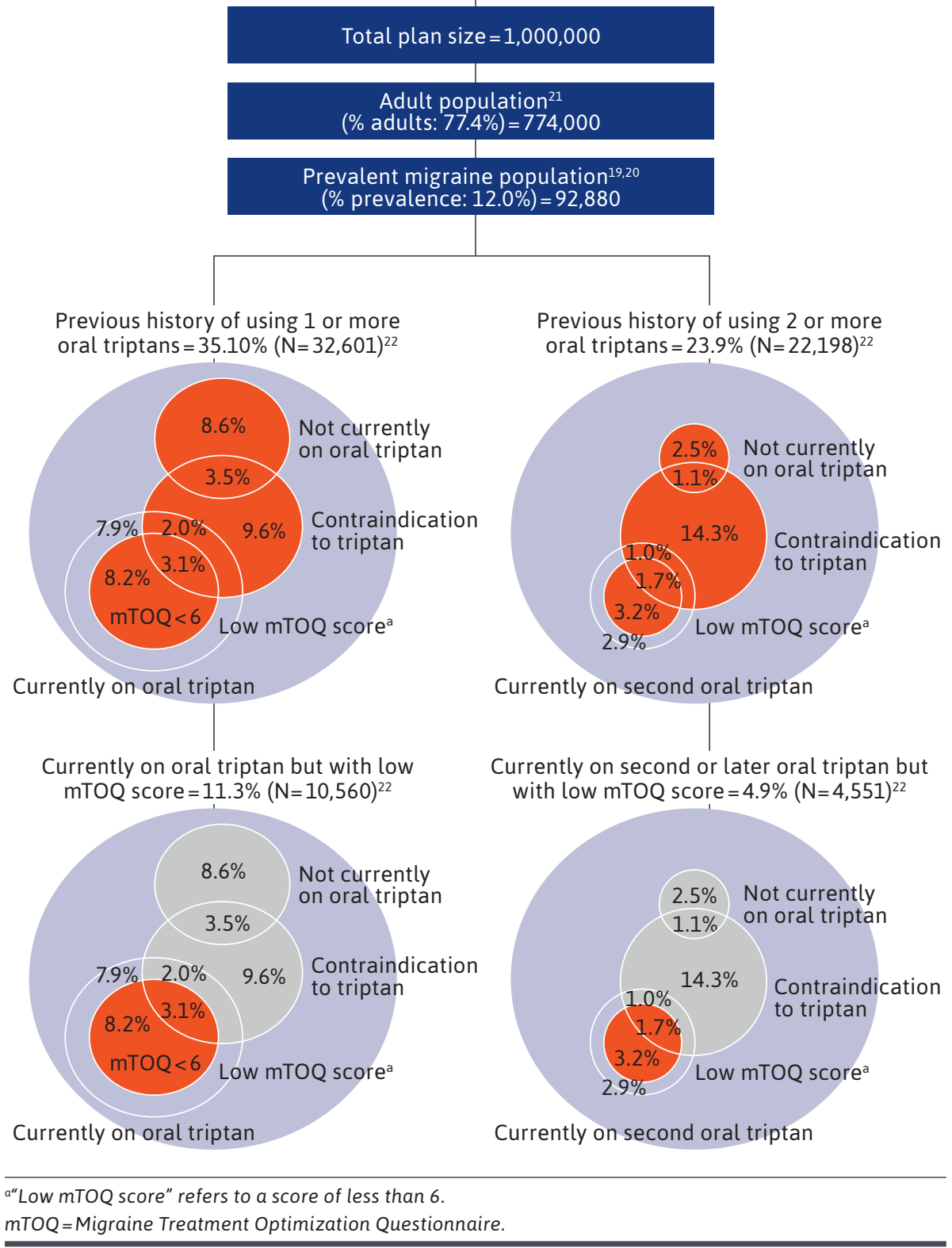

The model framework was developed from the perspective of a third-party commercial payer in the United States over a time horizon of 3 years. Primary outcomes were average cost per member per month (PMPM), total cumulative 3-year cost, cost per treated member per month, and the incremental difference between those outcomes in the scenario with and without lasmiditan.

The model was developed in accordance with guidelines and recommendations from the Academy of Managed Care Pharmacy and the International Society for
Pharmacoeconomics and Outcomes Research. ${ }^{20,21}$

\section{DATA INPUTS}

Population. Published epidemiological data were used to estimate the number of adults with migraine in the United States, ${ }^{22-24}$ accounting for annual population growth over 3 years. The size of the target population was obtained by estimating the percentage of adults with migraine who were eligible for the emerging novel acute treatments using the first cohort of OVERCOME US, a recent web-based survey. ${ }^{25}$ Baseline data for the initial OVERCOME US crosssectional survey were collected from September to November 2018 using a phased approach. First, a representative sample of respondents of the US population was sought using 5 commercial consumer panels. Inclusion criteria were (1) aged 18 years or older, (2) US resident, (3) online survey panel member, (4) internet access, (5) ability to read and write English, and (6) ability to provide electronic informed consent. Sample performance was monitored daily vs quota targets.

The sample of respondents $(\mathrm{N}=97,478)$ reflected the US marginal distribution of geography (Northeast, Midwest, South, and West); age (18-34, 35-44, 45-54, 55-64, and 65+ years); and race (American Indian/Alaska native, Asian/Asian American/Native Hawaiian/Asian or Pacific Islander, Black/African American, White/ Caucasian, and other), each nested in sex. OVERCOME US included 21,000 patients that met the criteria for migraine based on a validated American Migraine Study/AMPP migraine diagnostic screener or a self-report of migraine diagnosis by a health care professional.

The study considered 4 alternative criteria for the target populations for treatment (Figure 1). These criteria 


\section{TABLE 1}

Market Share Inputs as a Percentage of Target Population Over 3 Years and Non-Novel Acute Treatment at Baseline

\begin{tabular}{l|c|c}
\hline & $\begin{array}{c}\text { Population 1 } \\
\text { Previous history of } \\
\text { or more oral triptan } \\
\text { or contraindicated to } \\
\text { triptans }\end{array}$ & $\begin{array}{c}\text { Population 2 } \\
\text { Previous history of } \\
\text { 2 or more oral } \\
\text { triptans or } \\
\text { contraindicated to } \\
\text { triptans }\end{array}$ \\
\hline Novel treatments market uptake & \multicolumn{2}{|c}{} \\
\hline Year 1 & $2.6 \%$ & $1.9 \%$ \\
\hline Year 2 & $4.7 \%$ & $3.5 \%$ \\
\hline Year 3 & $8.0 \%$ & $5.9 \%$ \\
\hline Percentage of patients on non-novel treatment at baseline \\
\hline Oral triptans & $40.8 \%$ & $37.3 \%$ \\
\hline Non-oral triptans & $29.4 \%$ & $25.7 \%$ \\
\hline Ergots & $7.6 \%$ & $9.2 \%$ \\
\hline Prescription NSAIDs & $25.8 \%$ & $27.3 \%$ \\
\hline Opioids & $31.7 \%$ & $33.5 \%$ \\
\hline Barbiturates & $15.3 \%$ & $16.8 \%$ \\
\hline Antiemetics & $3.8 \%$ & $4.7 \%$ \\
\hline Antihistamines & $5.2 \%$ & $5.5 \%$ \\
\hline
\end{tabular}

continued on next page

probed eligibility requirements derived from the AHS position statement regarding use of the novel acute treatments comparing 1 vs 2 steps through oral triptans, as well as a comparison with a narrower subset of patients who are currently on an oral triptan but doing "poor" or "very poorly" on their current acute treatment (mTOQ score $<6$ ).

- Benefit-eligible populations

- Population 1: previous history of using 1 or more oral triptan or contraindicated to triptans.

- Population 2: previous history of using 2 or more oral triptans or contraindicated to triptans.

- "Most likely to use" populations

- Population 3: currently on oral triptan but with $\mathrm{mTOQ}<6$.

- Population 4: currently on second or later oral triptan but with $\mathrm{mTOQ}<6$.

The mTOQ-6 is a validated patient-reported questionnaire that focuses on how often over the past 3 months patients achieved treatment success in various domains: return to normal function, 2-hour pain freedom, sustained 24-pain relief, tolerability, comfort in being able to make plans, and perceived control. Since it was not anticipated that plans would have access to data such as mTOQ-6, this questionnaire was relevant to establishing the size of the population that fails to tolerate or receive adequate efficacy from current treatment. As such, the patients identified by using mTOQ-6 give insight on the size of the population that may be prescribed and submit claims for the novel acute treatments. ${ }^{26}$ Contraindications to oral triptans were based on medical history reported in OVERCOME US. ${ }^{25}$

Treatment Options. The market size of novel acute treatments for population 1 (previous history of using 1 or more oral triptans or contraindicated to triptans) was drawn from a hypothetical forecast scenario, whereas for population 2 (previous history of using 2 or more oral triptans or contraindicated to triptans), market size was estimated to be approximately $50 \%$ of that for population 1 . For the narrower populations (but thought to be the most likely to use), the market size for population 3 was assumed to be about $90 \%$ of that for population 1 , and for population 4 , about $90 \%$ of that for population 2 . The adoption of the novel agents within the plan was assumed to be independent of the number and mix of novel agents (lasmiditan, rimegepant, and ubrogepant) included in the formulary. Baseline market shares of the non-novel treatments were informed by the OVERCOME US survey (Table 1).

The distribution of market share among the 3 novel acute treatments was hypothetical based on preapproval market research estimates. In the scenario without lasmiditan, it was assumed that $50 \%$ were treated with ubrogepant and $50 \%$ were treated with rimegepant. When lasmiditan was included, the distribution was assumed to be $40 \%$, $30 \%$, and $30 \%$ for lasmiditan, ubrogepant, and rimegepant, respectively. Lasmiditan and ubrogepant are approved for use at more than 1 dose. The distribution over the 3 doses of lasmiditan was assumed to be $20 \%, 60 \%$, and $20 \%$ for the $50 \mathrm{mg}, 100 \mathrm{mg}$, and $200 \mathrm{mg}$ doses, respectively. The distributions were assumed to remain constant over the 3 -year time horizon.

Costs. The model was built from a US commercial payer perspective and considered only drug acquisition and administration costs. Indirect costs were not considered. Drug acquisition costs were based on wholesale acquisition costs obtained from Micromedex RED BOOK Online (October 2019 for non-novel treatments and March 2020 for novel treatments). ${ }^{27}$ Drug dosing and frequency were obtained from the prescribing information for each product, while the annual dosing frequency measured by the number of single-treatment doses per patient per year was informed by the MarketScan Commercial Claims and Encounters database, based on historical triptan data. ${ }^{28}$ 


\section{TABLE 1}

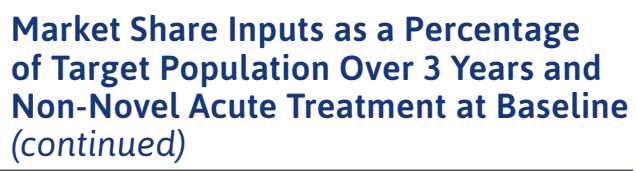

Market Share Inputs as a Percentage of Target Population Over 3 Years and Non-Novel Acute Treatment at Baseline (continued)

\begin{tabular}{l|c|c}
\hline \hline & $\begin{array}{c}\text { Population 3 } \\
\text { Currently on oral } \\
\text { triptan but with low } \\
\text { mTOQ score }\end{array}$ & $\begin{array}{c}\text { Population 4 } \\
\text { Currently on second } \\
\text { or later oral triptan } \\
\text { but with low mTOQ } \\
\text { score }\end{array}$ \\
\hline Novel treatments market uptake & \multicolumn{2}{|c}{} \\
\hline Year 1 & $7.1 \%$ & $8.2 \%$ \\
\hline Year 2 & $13.1 \%$ & $15.2 \%$ \\
\hline Year 3 & $22.1 \%$ & $25.7 \%$ \\
\hline
\end{tabular}

Percentage of patients on non-novel treatment at baseline ${ }^{\mathrm{b}}$

\begin{tabular}{l|c|c}
\hline Oral triptans & $107.1 \%$ & $107.2 \%$ \\
\hline Non-oral triptans & $69.4 \%$ & $63.4 \%$ \\
\hline Ergots & $11.3 \%$ & $19.5 \%$ \\
\hline Prescription NSAIDs & $49.9 \%$ & $57.2 \%$ \\
\hline Opioids & $56.8 \%$ & $61.3 \%$ \\
\hline Barbiturates & $29.6 \%$ & $39.3 \%$ \\
\hline Antiemetics & $8.2 \%$ & $12.1 \%$ \\
\hline Antihistamines & $10.1 \%$ & $11.4 \%$ \\
\hline
\end{tabular}

aThese percentages for market uptake should yield numbers of patients taking novel therapies that follow these rationales: (1) number of beneficiaries using novel acute treatments among those with previous history of using 1 or more oral triptan or contraindicated is drawn from a market forecast scenario; (2) number of beneficiaries using novel acute treatments among those with previous history of using 2 or more oral triptans or contraindicated is approximately $50 \%$ of scenario 1 ; (3) number of beneficiaries using novel acute treatments among those currently on oral triptan but with low MTOQ represents about $90 \%$ of scenario 1 ; and (4) number of beneficiaries using novel acute treatments among those currently on second or later oral triptan but with low $\mathrm{mTOQ}$ represents about $90 \%$ of scenario 2 .

${ }^{b}$ Given that a single patient can receive multiple treatments over the course of a year, the total patient treatment share in the model exceeds $100 \%$.

$m T O Q=$ Migraine Treatment Optimization Questionnaire; NSAID = nonsteroidal anti-inflammatory drug.

Based on analysis of insurance claims data for triptans, the annual number of treated migraine episodes was estimated to be 27 . The annual dosing frequency of novel acute treatments to treat the 27 episodes varied depending on the assumed use of rescue dosing. Almost $40 \%$ of ubrogepant patients in the phase 3 clinical studies used a rescue dose ${ }^{29,30}$ and $45 \%-48 \%$ used a rescue dose in the longerterm open label follow-up study. ${ }^{31}$ The model reflected this in the annual dosing frequency of ubrogepant. Conversely, labeled usage of lasmiditan and rimegepant precluded use of a second dose,, 1719 and their annual dosing frequency equaled 27 (Table 2). The $200 \mathrm{mg}$ dose of lasmiditan is available only as two $100 \mathrm{mg}$ pills. ${ }^{17}$ The cost per episode for lasmiditan $200 \mathrm{mg}$ was, therefore, taken as 2 times the cost per episode of $100 \mathrm{mg}$ of lasmiditan. The $50 \mathrm{mg}$ and $100 \mathrm{mg}$ doses of lasmiditan were priced the same on a per pill basis, as were the $50 \mathrm{mg}$ and $100 \mathrm{mg}$ doses of ubrogepant. ${ }^{27}$

\section{ANALYSIS}

We used the presented input values to calculate the annual and 3-year total costs for the 4 target populations, as well as the PMPM cost for the markets with and without lasmiditan. One-way sensitivity analyses were conducted to assess the effect of key parameters on the total cost impact in 3 years. Model parameters varied by plus or minus 15\% to assess uncertainty in model parameters and key model drivers. While plus or minus $15 \%$ may not precisely cover the plausible rage of each parameter, the variation does provide directional and approximate estimates of relative magnitude of impact.

\section{Results}

\section{BASE-CASE ANALYSES}

The addition of lasmiditan to a formulary alongside the other 2 novel acute agents ubrogepant and rimegepant resulted in a total savings over a 3-year time horizon of $-\$ 927,657$ (-1.5\%) in Population 1 (previous history of using 1 or more oral triptan or contraindicated to a triptan) and -\$830,796 (-1.8\%) in population 3 (currently on an oral triptan but with a $\mathrm{mTOQ}<6$ ). In population 2 (previous history of using 2 or more oral triptans or contraindicated), the addition of lasmiditan resulted in a total budget impact of -\$466,518 (-1.3\%); whereas in population 4 (currently using a second or later oral triptan but with $\mathrm{mTOQ}<6)$, the savings were $-\$ 415,601(-2.0 \%)$ over a 3-year time horizon (Table 3). Most of the cost savings was attributable to reductions in drug acquisition cost. Savings in total costs resulted in average incremental cost PMPM of $-\$ 0.01$ to -0.03 in the 4 target population groups. Detailed results may be found in Supplementary Table 1 (available in online article).

\section{SENSITIVITY ANALYSES}

Sensitivity analyses of the 4 target population groups were consistent (Figure 2). They suggested that drug acquisition costs of novel acute treatments, annual treated migraine episodes, and the uptake of novel treatments are key cost drivers. In addition, none of the tornado diagram bars crossed the line to positive incremental costs triggered by the introduction of lasmiditan to the formulary. 


\section{TABLE 2 Drug Acquisition and Administration Costs and Dosing Frequency}

\begin{tabular}{|c|c|c|c|c|}
\hline Treatments & Dosing & $\begin{array}{l}\text { Acquisition } \\
\text { cost per unit }\end{array}$ & $\begin{array}{c}\text { Annual dosing } \\
\text { frequency }\end{array}$ & Annual cost, \$ \\
\hline \multicolumn{5}{|l|}{ Novel treatments } \\
\hline Lasmiditan & $\begin{array}{l}20 \% \text { on } 50 \mathrm{mg} \\
60 \% \text { on } 100 \mathrm{mg} \\
20 \% \text { on } 200 \mathrm{mg}\end{array}$ & $\begin{array}{c}\$ 80 \text { per pill } \\
\text { (50 or } 100 \mathrm{mg}, 200 \mathrm{mg} \\
\text { dose }=2 \times 100 \mathrm{mg} \text { pills) }\end{array}$ & $\begin{array}{l}32.4 \\
(=27 \times 20 \%+27 \times \\
60 \%+54 \times 20 \%)\end{array}$ & 2,592 \\
\hline Ubrogepant & $\begin{array}{l}25 \% \text { on } 50 \mathrm{mg} \\
75 \% \text { on } 100 \mathrm{mg}\end{array}$ & $\begin{array}{c}\$ 85 \text { per pill } \\
\text { (50 or } 100 \mathrm{mg} \text { ) }\end{array}$ & $\begin{array}{c}38 \\
\qquad(=27 \times 1.4) \\
\text { Assume } 40 \% \text { of patients } \\
\text { using second dose as a } \\
\text { rescue medication }{ }^{\mathrm{b}}\end{array}$ & 3,230 \\
\hline Rimegepant & $100 \%$ on 75 mg ODT & $\$ 106.25$ per pill & 27 & 2,869 \\
\hline \multicolumn{5}{|l|}{ Non-novel treatment class } \\
\hline \multirow[t]{2}{*}{ Oral triptans } & Single-agent oral triptans & $\$ 3.72$ & 26 & 96.79 \\
\hline & Sumatriptan/naproxen & $\$ 26.96$ & 36 & 970.63 \\
\hline \multirow[t]{3}{*}{ Non-oral triptans } & Zolmitriptan (nasal) & $\$ 80.94$ & 18 & $1,456.92$ \\
\hline & Sumatriptan (nasal) & $\$ 49.96$ & 16 & 799.29 \\
\hline & Sumatriptan (SC) & $\$ 57.22$ & 6 & 343.35 \\
\hline \multirow[t]{2}{*}{ Ergots } & Dihydroergotamine (nasal) & $\$ 210.46$ & 8 & $1,683.71$ \\
\hline & Other ergotamine & $\$ 12.45$ & 36 & 448.09 \\
\hline Prescription NSAIDs & Diclofenac and others & $\$ 0.80$ & 57 & 45.74 \\
\hline \multirow[t]{2}{*}{ Opioids } & Butorphanol (nasal) & $\$ 7.25$ & 21 & 152.18 \\
\hline & Other opioids and combinations & $\$ 1.21$ & 37 & 44.68 \\
\hline Barbiturates & Butalbital and combinations & $\$ 2.60$ & 60 & 155.92 \\
\hline \multirow[t]{2}{*}{ Antiemetics } & Metoclopramide & $\$ 0.35$ & 30 & 10.40 \\
\hline & Prochlorperazine & $\$ 0.70$ & 30 & 21.09 \\
\hline Antihistamines & Promethazine & $\$ 0.47$ & 30 & 14.12 \\
\hline \multicolumn{5}{|l|}{ SC administration $\cos ^{\mathrm{c}}$} \\
\hline \multicolumn{2}{|l|}{ SC injection for sumatriptan } & $\$ 29.67$ & 6 & 178.02 \\
\hline \multicolumn{5}{|c|}{$\begin{array}{l}\text { bA second dose of ubrogepant } 50 \mathrm{mg} \text { and } 100 \mathrm{mg} \text { was taken by } 39.48 \% \text { (184/466) and } 40.82 \% \text { (198/485) of patients, respectively, in the ubrogepant group from the } \\
\text { ACHIEVE trial (ie, an average of } 40 \% \text { of all patients). This is reflected as an increase in the annual dosing frequency. } \\
\text { cFrom InHealth Professional Services. }{ }^{38} \\
\text { NSAID= nonsteroidal anti-inflammatory drug; ODT = orally disintegrating tablet; SC = subcutaneous. }\end{array}$} \\
\hline
\end{tabular}

\section{DISCUSSION}

The potential budget impact of the novel acute treatments has been explored by the Institute for Clinical and Economic Review (ICER). ${ }^{32,33}$ The target cohort used was similar to the cohorts examined here: patients for whom triptans had not been effective, were not tolerated, or were contraindicated. The ICER analysis was conducted at a US national level with results expressed as the proportion of patients that could be treated given an assumed total annual national expenditure of $\$ 819$ million and various costs per quality-adjusted life-year derived from ICER's cost-effectiveness modeling. The estimated proportions that could be treated without exceeding the budget threshold were $12 \%, 16 \%$, and $16 \%$, respectively, for lasmiditan, ubrogepant, and rimegepant.

At the time of the ICER analysis, the wholesale acquisition cost (WAC) for rimegepant was unknown and assumed to be the same as that for ubrogepant. Our model used the actual launch WAC for rimegepant $(\$ 106.25)$ per pill rather than that of ubrogepant (\$85 per pill). The ICER analysis pooled the various doses of lasmiditan and ubrogepant, 


\section{TABLE 3 Results Comparing Scenarios With and Without Lasmiditan Over 3 Years}

\begin{tabular}{|c|c|c|c|c|c|c|c|}
\hline \multirow[b]{2}{*}{ Target population } & \multicolumn{2}{|c|}{$\begin{array}{l}\text { Scenario without } \\
\text { lasmiditan }\end{array}$} & \multicolumn{2}{|c|}{$\begin{array}{l}\text { Scenario with } \\
\text { lasmiditan }\end{array}$} & \multicolumn{3}{|c|}{ Incremental results } \\
\hline & Total cost, \$ & PMPM, \$ & Total cost, \$ & PMPM, \$ & $\begin{array}{l}\text { Incremental } \\
\text { total costs, \$ }\end{array}$ & $\begin{array}{l}\text { Incremental } \\
\text { PMPM, \$ }\end{array}$ & $\%$ change \\
\hline $\begin{array}{l}\text { Previous history of using } 1 \text { or more oral triptans or } \\
\text { contraindicated to triptans }\end{array}$ & $60,306,449$ & 1.65 & $59,378,793$ & 1.63 & $-927,657$ & -0.03 & -1.5 \\
\hline $\begin{array}{l}\text { Previous history of using } 2 \text { or more oral triptans or } \\
\text { contraindicated to triptans }\end{array}$ & $37,126,026$ & 1.01 & $36,659,509$ & 1.00 & $-466,518$ & -0.01 & -1.3 \\
\hline Currently on oral triptan but with low mTOQ score & $46,004,093$ & 1.26 & $45,173,296$ & 1.24 & $-830,796$ & -0.02 & -1.8 \\
\hline $\begin{array}{l}\text { Currently on second or later oral triptan but with } \\
\text { low mTOQ score }\end{array}$ & $21,172,170$ & 0.58 & $20,756,569$ & 0.57 & $-415,601$ & -0.01 & -2.0 \\
\hline
\end{tabular}

mTOQ = Migraine Treatment Optimization Questionnaire; $P M P M=$ per member per month.

and as discussed further in this section, did not distinguish between the differences in label-approved usage for a second dose within a single episode of migraine.

Our model concluded that the introduction of lasmiditan alongside oral CGRP receptor antagonists as an option for the treatment of migraine with and without aura in adults resulted in a decrease in a US commercial payer's budget over a 3-year time horizon. The acquisition cost per pill difference is smaller between lasmiditan and ubrogepant (\$80 vs \$85), but this may not reflect the full drug cost/ migraine attack, since a second dose of ubrogepant may be administered if needed. Approximately $40 \%$ of ubrogepant patients in the phase 3 clinical studies used a rescue dose. On the other hand, two $100 \mathrm{mg}$ pills of lasmiditan are required to achieve the $200 \mathrm{mg}$ dose. It is not yet known what proportion of patients will opt to use the $200 \mathrm{mg}$ dose of lasmiditan at the higher cost per episode.

US commercial plans may implement a variety of designs regarding use of the novel acute treatments. The AHS guidance suggests a step through 2 or more oral triptans if not contraindicated; however, plans may choose to give access after only 1 step to avoid their beneficiaries delaying trial of a treatment with a different mechanism of action. Switching from one triptan to another is not associated with significant improvements in headache-related disability compared with consistent treatment. ${ }^{34}$ Furthermore, inadequate acute treatment efficacy was associated with an increased risk of new-onset chronic migraine over the course of 1 year. ${ }^{35}$

In addition to benefit eligible populations, the model explored 2 smaller populations of patients thought to be more likely to receive therapy. These populations were based on patients currently receiving an oral triptan but reporting a low mTOQ $(<6)$ score, indicating that they were not adequately treated. While considerably smaller in terms of the number of beneficiaries, these populations were thought to have a high likelihood of receiving the novel acute treatments and in the end account for approximately $90 \%$ of the total market of the larger benefit eligible populations. Future analyses of claims data will be needed to assess the number and characteristics of beneficiaries who actually initiate the novel acute treatments.

The univariate sensitivity analyses suggested that drug acquisition costs of novel acute treatments, annual treated migraine episodes, and the uptake of novel treatments were the most influential parameters on results. The addition of lasmiditan maintained cost savings even if the costs of the novel acute medications were decreased by $15 \%$.

This study drew heavily on US real-world data. The size of the eligible population and market shares of current treatments were based on data from the OVERCOME US study, whereas the dosing frequencies were based on information from the MarketScan claims database.

\section{LIMITATIONS}

As with all economic analyses, several limitations should be noted. First, the generalizability of all insurance plans will depend on how well data sources and assumptions represent the plan members' patterns of migraine management and associated medical costs. As noted, use of data from a representative US patient survey was likely a plus for this analysis.

Second, the model was developed for cost comparison only and did not consider product safety, efficacy, or outcomes. As such, uptake of novel therapies by eligible patients was not adjusted for any potential differences between the agents on these attributes. The label for lasmiditan includes these warnings: Patients are advised not 
FIGURE 2 One-Way Sensitivity Analysis: Impact of Changes of Input Parameters $( \pm 15 \%)$ on the Change in Total Cost in 3 Years (Target Population: Patients With Previous History of 1 or More Oral Triptans)

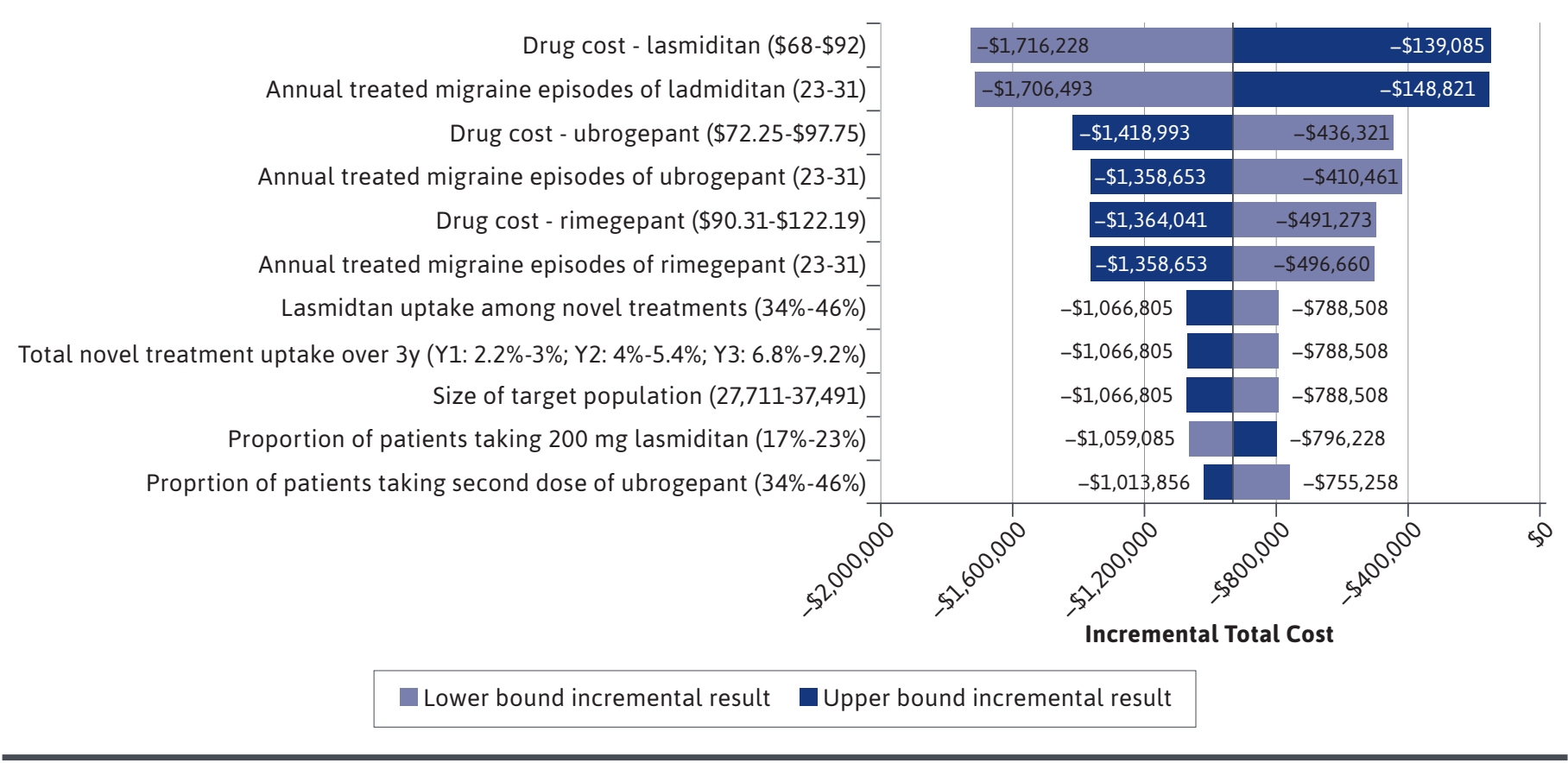

to drive or operate machinery until at least 8 hours after taking each dose of lasmiditan. Patients may not be able to assess their own driving competence and the degree of impairment. Lasmiditan may cause central nervous system (CNS) depression and should be used with caution if used in combination with alcohol or other CNS depressants. Reactions consistent with serotonin syndrome were reported in patients treated with lasmiditan, and patients should discontinue lasmiditan if symptoms of serotonin syndrome occur. Detoxification may be necessary in case of medication overuse headaches.

The label for rimegepant warns that if a serious hypersensitivity reaction occurs, patients are to discontinue rimegepant and initiate appropriate therapy. Severe hypersensitivity reactions have included dyspnea and rash and can occur days after administration.

In GLADIATOR, ${ }^{36,37}$ the long-term open-label extension study of lasmiditan, more patients discontinued because of adverse events than in ubrogepant and rimegepant longterm extension studies. The most common adverse event leading to discontinuation with lasmiditan was dizziness. Discontinuation because of dizziness was dose dependent and more likely to occur after the first or second treated attack than after subsequent attacks, suggesting the possibility that the impact may be mitigated in clinical practice by dose adjustment, which was not permitted in the trial.

Third, the model did not include the costs related to health care resource utilization (HCRU). The phase 3 registration studies for the 3 drugs were based on single episodes of care and did not include longer-term evaluations of resource utilization. We chose not to speculate on HCRU until more definitive data were available. We also did not consider patient cost sharing (ie, copayment or coinsurance); pharmaceutical incentives (ie, rebates); or dispensing fees, since this would introduce complexity related to shifts between medical and pharmacy benefits due to changes in care locations and routes of administration and would in the end have minimal effect on the analysis.

Finally, these analyses assumed the distribution for the 3 doses of lasmiditan $(20 \%$ on $50 \mathrm{mg}, 60 \%$ on $100 \mathrm{mg}$, and $20 \%$ on $200 \mathrm{mg}$ ) and of the proportion of patients on ubrogepant taking a second dose $(40 \%$ based on a phase 3 clinical trial). Budget impact results may change if these proportions differ from real-world practice. 


\section{Conclusions}

The addition of lasmiditan to the formulary as a novel acute treatment option for migraine alongside ubrogepant and rimegepant resulted in lower budget impact from a US commercial payer perspective.

\section{DISCLOSURES}

This work was funded by Eli Lilly and Company. Milev and Sun are employed by Evidera, which received funding from Eli Lilly and Company for work on this project. Pohl, Mason, Njuguna, and Loo are employees and stockholders of Eli Lilly and Company.

\section{REFERENCES}

1. Headache Classification Committee of the International Headache Society (IHS). The International Classification of Headache Disorders, 3rd edition. Cephalalgia. 2018;38(1):1-211.

2. Woldeamanuel YW, Cowan RP. Migraine affects 1 in 10 people worldwide featuring recent rise: A systematic review and meta-analysis of community-based studies involving 6 million participants.

J Neurol Sci. 2017;372:307-315.

3. Lipton RB, Stewart WF, Diamond S, Diamond ML, Reed M. Prevalence and burden of migraine in the United States: data from the American Migraine Study II. Headache. 2001;41(7):646-57.

4. Burch RC, Loder S, Loder E, Smitherman TA. The prevalence and burden of migraine and severe headache in the United States: updated statistics from government health surveillance studies. Headache. 2015;55(1):21-34.

5. Burshtein R, Burshtein A, Burshtein J, Rosen N. Are episodic and chronic migraine one disease or two? Curr Pain Headache Rep. 2015;19(12):53.

6. American Migraine Foundation. Living with migraine. 2018. Accessed March 12, 2018. https://americanmigrainefoundation.org/living-with-migraines/
7. Blumenfeld AM, Varon SF, Wilcox TK, et al. Disability, HRQoL and resource use among chronic and episodic migraineurs: results from the International Burden of Migraine Study (IBMS). Cephalalgia. 2011;31(3):301-15.

8. Raval AD, Shah A. National trends in direct health care expenditures among US adults with migraine: 2004 to 2013. J Pain. 2017;18(1):96-107.

9. Bonafede M, Sapra S, Shah N, Tepper S, Cappell K, Desai P. Direct and indirect healthcare resource utilization and costs among migraine patients in the United States. Headache. 2018;58(5):700-14.

10. Messali A, Sanderson JC, Blumenfeld AM, et al. Direct and indirect costs of chronic and episodic migraine in the United States: a web-based survey. Headache. 2016;56(2):306-22.

11. Silberstein SD. Practice parameter: evidence-based guidelines for migraine headache (an evidence-based review): report of the Quality Standards Subcommittee of the American Academy of Neurology. Neurology. 2000;55(6):754-62.

12. Marmura MJ, Silberstein SD, Schwedt TJ. The acute treatment of migraine in adults: the American Headache Society evidence assessment of migraine pharmacotherapies. Headache. 2015;55(1):3-20.

13. Lipton RB, Buse DC, Serrano D, Holland S, Reed ML. Examination of unmet treatment needs among persons with episodic migraine: results of the American Migraine Prevalence and Prevention (AMPP) Study. Headache. 2013;53(8):1300-11.

14. Diener HC, Limmroth V. Advances in pharmacological treatment of migraine. Expert Opin Investig Drugs. 2001;10(10):1831-45.

15. Dodick DW. Triptan nonresponder studies: implications for clinical practice. Headache. 2005;45(2):156-62.
16. American Headache Society. The American Headache Society position statement on integrating new migraine treatments into clinical practice. Headache. 2019;59(1):1-18.

17. REYVOW (lasmiditan). Prescribing information. Lilly USA; 2020. Revised August 2021. Accessed November 12, 2020. http://pi.lilly.com/us/reyvow-uspi.pdf

18. UBRELVY (ubrogepant). Prescribing information. Allergan; 2019. Accessed February 14, 2020. https://www. accessdata.fda.gov/drugsatfda_docs/ label/2019/211765s000lbl.pdf

19. NURTEC ODT (rimegepant). Prescribing information. Biohaven Pharmaceuticals; 2020. Revised May 2021. Accessed February 28, 2020. https:// www.nurtec.com/pi

20. Foundation for Managed Care Pharmacy and Academy of Managed Care (FMCP) Format Executive Committee. The AMCP format for formulary submissions version 4.1. April 20, 2020. Accessed November 15, 2021. https:// www.amcp.org/Resource-Center/ format-formulary-submissions/AMCPFormat-for-Formulary-Submissions-4.1

21. Caro JJ, Briggs AH, Siebert U, Kuntz KM, Force I-SMGRPT. Modeling good research practices-overview: a report of the ISPOR-SMDM Modeling Good Research Practices Task Force-1. Med Decis Making. 2012;32(5):667-77.

22. Lipton RB, Bigal ME, Diamond M, et al. Migraine prevalence, disease burden, and the need for preventive therapy. Neurology. 2007;68(5):343-49.

23. Buse DC, Manack AN, Fanning KM, et al. Chronic migraine prevalence, disability, and sociodemographic factors: results from the American Migraine Prevalence and Prevention Study. Headache. 2012;52(10):1456-70.

24. US Census Bureau. Quick facts table. Accessed March 12, 2019. https://www. census.gov/quickfacts/fact/table/US/ $\underline{\text { PST045218 }}$ 
25. Lipton RB, Pohl GM, Araujo AB, et al. Who is eligible for novel medications designed for the acute treatment of migraine and what are their unmet needs? Results of the OVERCOME Study. [abstract]. Headache. 2019;59(S1):57-58. doi: 10.1111/head.13549

26. Serrano D, Buse DC, Manack Adams A, Reed ML, Lipton RB. Acute treatment optimization in episodic and chronic migraine: results of the American Migraine Prevalence and Prevention (AMPP) Study. Headache. 2015;55(4):502-18.

27. IBM. IBM Micromedex RED BOOK. Database. Accessed October 2019. https://www.ibm.com/products/ micromedex-red-book

28. IBM Watson Health. IBM MarketScan Research Databases for life sciences researchers. White paper. 2018. Accessed November 15, 2021. https://www.ibm. com/downloads/cas/0NKLE57Y

29. Dodick DW, Lipton RB, Ailani J, et al. Ubrogepant for the treatment of migraine. N Engl J Med. 2019;381(23):2230-41.
30. Lipton RB, Dodick DW, Ailani J, et al. Effect of ubrogepant vs placebo on pain and the most bothersome associated symptom in the acute treatment of migraine: the ACHIEVE II Randomized Clinical Trial. JAMA. 2019;322(19):1887-98.

31. Ailani J, Lipton RB, Hutchinson S, et al. Long-term safety evaluation of ubrogepant for the acute treatment of migraine: phase 3, randomized, 52-week extension trial. Headache. 2020;60(1):141-52.

32. Atlas S TD, Agboola F, Lee T, Chapman R, Pearson S D, Rind DM,. Acute treatments for migraine: effectiveness and value. Institute for Clinical and Economic Review. 2020. Accessed February 25, 2020. http://icer-review.org/material/ acute-migraine-evidence-report/

33. Agboola F, Atlas SJ, Touchette DR, Borrelli EP, Rind DM, Pearson SD. The effectiveness and value of novel acute treatments for migraine. J Manag Care Spec Pharm. 2020;26(11):1456-62. doi: 10.18553/jmcp.2020.26.11.1456

34. Serrano D, Buse DC, Kori SH, et al. Effects of switching acute treatment on disability in migraine patients using triptans. Headache. 2013;53(9):1415-29.
35. Lipton RB, Fanning KM, Serrano D, Reed ML, Cady R, Buse DC. Ineffective acute treatment of episodic migraine is associated with new-onset chronic migraine. Neurology. 2015;84(7):688-95.

36. Brandes JL, Klise S, Krege JH, et al. Interim results of a prospective, randomized, open-label, Phase 3 study of the long-term safety and efficacy of lasmiditan for acute treatment of migraine (the GLADIATOR study). Cephalalgia. 2019;39(11):1343-57.

37. Brandes JL, Klise S, Krege JH, et al. Long-term safety and efficacy of lasmiditan for acute treatment of migraine: final results of the GLADIATOR study. Cephalalgia Reports. Published online September 15, 2020. doi:10.1177/2515816320958176

38. InHealth Professional Services. 2019 Physicians' fee \& coding guide. Commercial fee range. 2019. Accessed October 2019. https://store.coderscentral. com/storefrontCommerce/subcategorybrowse.do? subcategory-name $=$ Fees $+\% 26+$ RVUs+Products\&path $=$ CodersCentral 